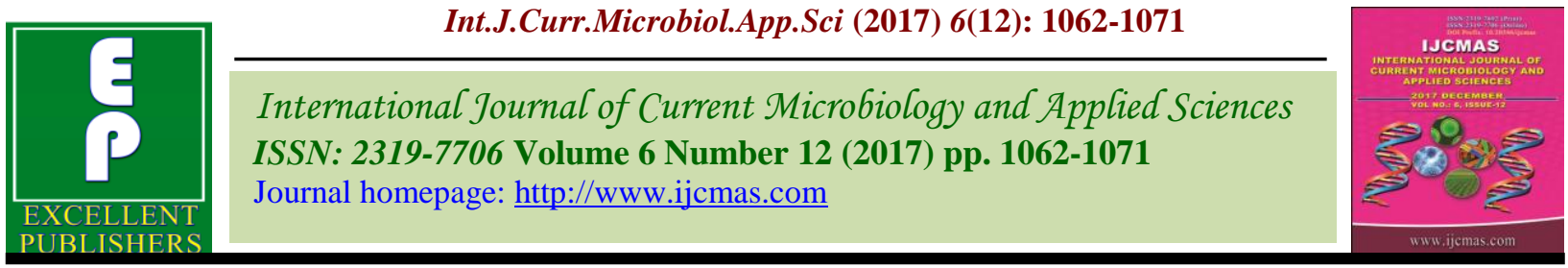

Review Article

https://doi.org/10.20546/ijcmas.2017.612.120

\title{
Isoflavones: Biosynthesis, Health Promoting Effects and Bioavailability
}

\author{
Sandeep Kumar* \\ ICAR-IARI, New Delhi-110012, India \\ *Corresponding author
}

\begin{abstract}
A B S T R A C T
\end{abstract}

\begin{tabular}{|c|c|}
\hline $\begin{array}{l}\text { Ke y w o r d s } \\
\text { Biosynthesis, } \\
\text { Health, } \\
\text { Bioavailability. }\end{array}$ & $\begin{array}{l}\text { Isoflavones are one of the various phytochemicals present in soybean considered } \\
\text { responsible for its health benefits. Isoflavone come under the class of polyphenolic } \\
\text { compounds and are of different chemical forms. In soybean genistein, daidzein and their } \\
\text { glycosidic forms are the major isoflavones present. In addition to these small amounts of } \\
\text { glycitein and its derivatives are also present. A branch of phenyl propanoid pathway is }\end{array}$ \\
\hline Article Info & key enzyme of this pathway. After action of this enzyme other modifications like \\
\hline $\begin{array}{l}\text { Accepted: } \\
10 \text { October } 2017 \\
\text { Available Online: } \\
10 \text { December } 2017\end{array}$ & $\begin{array}{l}\text { glycosylation and malonylation can occur. Due to its structural similarity to estrogens, } \\
\text { isoflavones show agnostic and sometimes antagonistic activity, which is considered } \\
\text { responsible for anti-cancerous properties of soybean. However, bioavailability is a key } \\
\text { factor for bioactivity. The use of microbial fermentation/isolation of efficient hydrolyzing } \\
\text { enzymes can make the isoflavones more bioavailable in human digestive system. }\end{array}$ \\
\hline
\end{tabular}

\section{Introduction}

Soybean is the most important legume crop grown around the globe. It is believed to have first domesticated in China around eleventh century B.C. or perhaps a bit earlier. It is used mainly for edible oil extraction, animal feed and food purpose. Soybean seed contains about $18 \%$ oil, $38 \%$ protein and $15 \%$ each of soluble and insoluble carbohydrates (http://www.nrcsoya.nic.in/soyfood.htm). Its oil contains a large proportion $(61 \%)$ of polyunsaturated fatty acids (PUFAs) about $15 \%$ saturated and $24 \%$ monounsaturated fatty acid. Among the PUFAs 55\% is linoleic acid while the linolenic acid content is about $10 \%$. Soybean oil has omega 3/omega 6 fatty acids ratio around 1:7.5 which is significantly higher than other vegetable oils. Due to high amount of PUFAs soybean oil is effective in lowering the blood cholesterol level and low density lipoprotein thus is good for heart patients. It also contains high quantity of vitamin $\mathrm{E}$ (tocopherols) which is helpful in coronary heart diseases and cancer and possibly acts through synergistic mechanism along with other phytochemicals (Rui, 2004). High biological value and PDCAAS (protein digestibility corrected amino acid score) make soy protein nutritionally comparable to animal proteins like casein, egg and beef (Sarwar, 1989).

Phytochemicals are the naturally occurring plant chemicals that have biological effects. Soybean has a high quantity of phytochemicals such as isoflavones, tocopherols, saponins, phytosterols and 
lecithin. Owing to the presence of these phytonutrients several health benefits such as reduction in heart diseases instances, hormone dependent cancers like breast cancer and osteoporosis have been associated with consumption of soybean. However, most of these beneficial effects have been correlated with significantly high levels of isoflavones (Dastmalchi and Dhaubhadel, 2015).

Isoflavones are a class of secondary metabolites whose production is limited to Papilionaceae family. In isoflavones the position of one aromatic ring is shifted. While most flavonoids have $\mathrm{B}$ ring attached to position 2 of $\mathrm{C}$ ring, isoflavones have $\mathrm{B}$ ring attached to position 3 of $\mathrm{C}$ ring (Fig. 1). Major Isoflavones present in soybean are genistein, daidzein and glycitein. They conjugate with sugars to form $\square \square$ glucosides (genistin, daidzin), which in turn can be acylated to form 6"-O-malonyl glucosides and 6"-O-acetyl glucosides etc. In plants, isoflavones occur predominantly in these conjugated forms and aglycones are present only in small amounts. In soybean, isoflavone content and composition vary depending upon variety, processing etc. (Tanahindarto et al., 2013).

\section{Biosynthesis}

Isoflavones are synthesized via a branch of phenylpropanoid pathway starting from Phenylalanine Ammonia Lyase (PAL) and ending with isoflavone synthase. Isoflavone synthase (IFS) is a cytochrome P450 monooxygenase bound to endoplasmic reticulum is the ultimate enzyme involved in the pathway. In soybean, two isoforms of this enzyme are present (Jung et al., 2000). An increased activity of IFS may cause increased generation of isoflavones because it is the key regulatory enzyme involved in isoflavone production (Pregelj et al., 2010). The isoflavone synthase was shown to be carrying out isoflavone biosynthesis from flavanone via hydroxylation coupled to aryl migration (Steele et al., 1999). Naringenin and liquiritigenin (both flavanones) act as substrate for isoflavone synthase and result in formation of genistein and daidzein respectively. Glucosyl and malonyltransferases often act on these aglycones to form conjugates with glucosyl and malonyl residues. In soybean glycosidic forms are abundant while the aglycones are found only in small amount. Two genes encoding isoflavone synthase were identified from soybean exploiting the nature of enzyme as a protein of cytochrome P450 family by screening yeast ESTs of soybean and expressed it in Arabidopsis resulting in production of isoflavones in A. thalina which otherwise doesn't synthesise isoflavones (Jung et al., 2000). This further established the role of IFS as a key enzyme in isoflavone synthesis. Dhaubhadel et al., (2008) identified the glycosyltranferase (UGT73F2) and malonyltransferase (GmMT7) in soybean which are responsible for formation of glycosyl- and malonyl- conjugates respectively. Both of these enzymes were found be localized in cytoplasm. Suzuki et al., (2007) cloned and characterized a cDNA encoding malonyl-CoA: isoflavone 7-Oglucoside-6"-O-malonyltransferase an enzyme catalyzing malonyl transfer to isoflavone 7-O- $\square$-D-glucosides to produce corresponding isoflavone 7-O-(6"-O-malonyl$\square$-D-glucoside).

\section{Factors influencing isoflavone accumulation in soybean seeds}

Soybean seeds have total isoflavone content in a range from $0.05 \%$ to $0.5 \%$ of the dry matter. Although individual isoflavones vary within this range in soybean seeds but it is well established that genistein, daidzein and their conjugates are present in high and nearly equal amount. Glycitein and its conjugates on 
the other hand are present in lesser amount (Lee et al., 2004). Several factors influence the isoflavone content and composition in soybean like genotype, location and year of seeding. Due to the potential health promoting effects of isoflavones several studies have been done for determining the isoflavone profile so that genotypes having better health promoting effects can be selected. $\mathrm{Xu}$ and Chang (2008) studied the isoflavone content in 30 American soybean genotypes. They found that the total isoflavone content varied from $1.18 \mathrm{mg} / \mathrm{g}$ to $2.86 \mathrm{mg} / \mathrm{g}$. Among different isoflavones genistein and it conjugates were most abundant (69\%). Wang and Murphy (1994) studied the impact of seeding year upon isoflavone profile and observed a significant effect of seeding year upon isoflavone content but such a relationship was absent for seeding location. However, Hoeck et al., (2000) reported that location of seeding can have an impact on the isoflavone accumulation in soybean. In an conclusive study Lee et al., (2003) reported variation of isoflavone content in 15 soybean genotypes with a range of 2.20 to $6.45 \mathrm{mg} / \mathrm{g}$ in the year 1998, 3.19 to $9.5 \mathrm{mg} / \mathrm{g}$ in the year 1999 and in the year 2000 isoflavone content varied from 2.93 to $4.83 \mathrm{mg} / \mathrm{g}$. They also observed that year of seeding tends to influence isoflavone content more as compared to genotype and location differences. Effects of location and seeding year can in part be explained by temperature differences due to planting date or those occurring during seed development.

Dhaubhadel et al., (2003) studied the isoflavonoid biosynthesis and accumulation in developing seeds and found that developing soybean embryos have an ability to synthesize isoflavonoids de novo but transport from maternal tissue may in part also contribute to isoflavonoid accumulation in seed. Also the isoflavone synthase 2 (IFS2) was found to be induced upon pathogen infection, UV light and its expression levels correlated well with the isoflavone accumulation while the IFS1 (another isoform) was expressed in an almost ubiquitous manner. Kudou et al., (1991) reported that in the mature seeds isoflavone accumulate to the greatest extent in hypocotyls and were lowest in seed coat Isoflavone synthase transcripts were detected primarily in the roots and seeds. The expression pattern of IFS1 in soybean was found to be consistent with the physiological roles of isoflavonoids as defence compounds against pathogens and signal molecules to symbiotic bacteria in soybean (Subramanian et al., 2004). Isoflavone level in soybean was found to be altered by phytochrome mediated light treatments (Kirakosyan et al., 2006). Isoflavone content was found to decrease upon drought during seed development (Gutierrez-Gonzalez et al., 2010) similar results were obtained for high temperature stress (Chennupati et al., 2012). Dastmalchi et al., (2016) studied the subcellular localization and interactions of major enzymes involved in isoflavone biosynthesis.

Isoflavone synthase, the key enzyme involved in isoflavone biosynthesis and cinnamate 4hydroxylase (both P450 enzymes) were found to be anchored to endoplasmic reticulum (ER). These enzymes were also found to be interacting with the soluble enzymes involved in phenylpropanoid pathway and isoflavone biosynthesis viz. chalcone synthase, chalcone reductase and chalcone isomerase. Through such interactions these soluble enzymes are tethered to the endoplasmic reticulum.

Interactions between soluble enzymes also have a role to play in maintaining this protein complex. All these findings point towards the existence of protein complex (isoflavonoid metabolon) mediating metabolic flux. Another interesting finding of the same study is the interaction of $\mathrm{GmCHR} 14$ only with the GmIFS2 but not with GmIFS1 (both 
catalyzing same reaction). GmCHR14 is responsible for the production of isoliquiritigenin (metabolite specific only to legumes). Isoliquiritigenin is acted upon by IFS to convert it into daidzein which can further produce phytoalexins. This interaction thus might be playing an important role under stress conditions.

In most of the non-leguminous plants precursor of isoflavone biosynthesis pathway i.e. flavanones are present but absence of IFS makes these plants unable to accumulate isoflavones. Many efforts have been made for accumulating isoflavones in non-leguminous plants but with limited success possibly because substrate is not present in sufficient amount to create sufficient amount of flux (Shih et al., 2008; Tian and Dixon, 2006; Jung et al., 2000; Yu et al., 2000). Pandey et al., (2014) made an effort for co-expression of AtMYB12, an Arabidopsis transcription factor which up-regulates genes of phenyl propanoid pathway viz. CHS, CHI, etc. and GmIFS1 genes in tobacco. Expression of these genes resulted in accumulation of substantial amount of genistein glycoconjugates. Treatment with these transgenic plants showed improved osteoprotective effect in Oestrogen-deficient mice.

\section{Biological roles of isoflavones in plants}

Isoflavones are found almost exclusively in legumes and have several different biological activities (Veitch, 2007). In legumes, isoflavones mediate plant-microbe interaction, induce Nod gene of nitrogen fixing bacteria. Subramanian et al., (2006) provided direct genetic evidence that isoflavones are essential for nodulation of soybean roots, due to their ability to stimulate nod genes present in Bradyrhizobium japonicum. They also observed that although isoflavones have an ability to modulate polar transport of auxin but isoflavones are not necessary for nodulation of soybean roots. Isoflavones also serve as precursors of more complex phytoalexins involved in plant defence. Simpler isoflavones like daidzein and genistein themselves also have antimicrobial activity. So it comes as no surprise that isoflavone biosynthesis is induced by biotic elicitors i.e. Jasmonic acid and salicylic acid (Farag et al., 2008; Shinde et al., 2009).

Fig.1 Isoflavone backbone

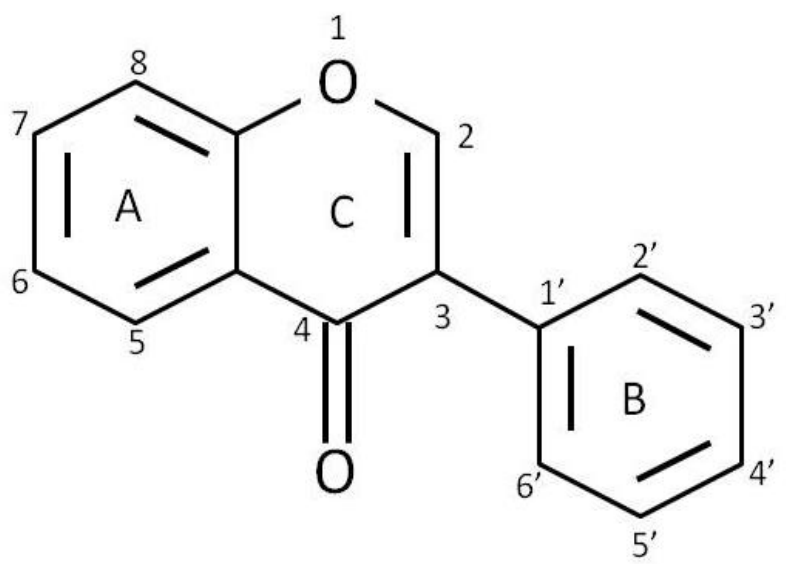




\section{Health promoting effects of isoflavones}

Lower incidences of oxidation linked diseases in soybean eating populations in comparison to Western countries was shown to be caused due to presence of antioxidant compounds like isoflavones (Sarkar and Li., 2003) in soybean. Cassidy et al., (1994) observed that isoflavones decrease incidence of hormonedependent breast and prostate cancers and osteoporosis due to structural similarity of isoflavone to estrogens because of which they can interfere with binding of estrogens to receptors and thus increase follicular phase length and/or delay menstruation. In a review of studies conducted to know efficacy of soy in improving symptoms of breast cancer; Fritz et al., (2013) found that higher soy consumption, may have protective function against the breast cancer development, mortality as well as recurrence. Protective effects of genistein in prostate cancer are attributed to epigenetic changes occurring via histone acetylation, demethylation and chromatin remodelling and thus causing activation of tumor suppressor genes (Majid et al., 2008; Kikuno et al., 2008). Isoflavones have ability to bind to both $\square$ and $\square$ type of estrogenic receptors especially to the receptors. Depending on the level of endogenous estrogen present and tissue type the effect of isoflavones can be estrogenic or anti-estrogenic (Kuiper et al., 1998; Kuiper et al., 1997). In case of reproductive tissues i.e. breast, prostate, uterus the risk of hormone dependent cancer decreases due to antiestrogenic effects of isoflavones. In nonreproductive tissues isoflavones have preventative effects towards hypercholesterolemia and osteoporosis due to estrogenic effects. Isoflavones show weak estrogenic activity i.e. $10^{-2}$ to $10^{-4}$ times as that of $17 \square$-estradiol. Among genistein and daidzein the estrogenic activity of genistein is ten times more as compared to daidzein. Isoflavones also show biological activity in ways other than their interaction with estrogenic receptors e.g. Genistein inhibit 3 and $17 \square$ hydroxysteroid activity and suppresses the synthesis of enzymes involved in estrogen metabolism (Whitehead et al., 2002). Genistein also inhibits the tyrosine kinase activity of epidermal growth factor (EGF) receptor (Akiyama et al., 1987). Obesity is considered as a major risk factor involved in the development of breast cancer. Lifelong exposure to isoflavones causes a decrease in chances to develop obesity in female rats which in turn reduces the risk for development of breast cancer (Kurrat et al., 2016). Dong et al., (2013) treated prostate cancer cells with different concentrations of genistein, daidzein $(25-200 \mathrm{mM})$ and combination of these at a concentration 25 or $50 \mathrm{mM}$. A low dose combination $(25 / 50 \square \mathrm{M}$ daidzein and $50 \quad \square \mathrm{M}$ genistein) showed synergistic effect on inducing apoptosis while only the $50 \square \mathrm{M}$ daidzein $+50 \square \mathrm{M}$ genistein combination had an impact on cell cycle. Also the daidzein was poorly taken up by prostate cancer cells than genistein.

\section{Isoflavones as antioxidants}

Pratt and Birac (1979) reported that antioxidant activities of soybeans can largely be attributed to isoflavones. Among various isoflavones genistein was found to be most potent antioxidant, both in the aqueous and in the lipophilic phases (Ruiz et al., 1997). However, isoflavones undergo extensive metabolism in the gut and liver, which might affect their biological properties, 8hydroxydaidzein was found to be most potent scavenger of hydroxyl and superoxide anion radicals. Isoflavone metabolites also exhibited higher antioxidant activity than parent compounds in standard antioxidant (FRAP and TEAC) assays (Rimbach et al., 2003). Patel et al., (2001) reported that isoflavones inhibit lipid peroxidation stimulated in both metal dependent and independent manner. 
However, isoflavones remained in native state and were not consumed which is inconsistent with classical antioxidants involved in chain terminating peroxy radical scavenging reactions. Antioxidant activity makes a major contribution towards the ability of isoflavones to reduce the risk of colon cancer (Mac Donald et al., 2005) and cardiovascular diseases (Diplock, 1994; Rice -Evans et al., 1997). Wiseman et al., (2000) investigated the possible effects of soy-isoflavone rich diet on the lipid peroxidation and LDL oxidation by comparing it with a diet containing soybean from which isoflavones were extracted. They observed that consumption of soybean containing isoflavones increased the LDL's resistance towards oxidation and reduced the amount of lipid peroxidation. Djuric et al., (2001) studied the effect of soy isoflavone supplementation on oxidative damage in blood and observed a decrease in level of oxidative damage to DNA in human and suggested that this may be one of the mechanisms responsible for cancer preventative properties of isoflavones.

\section{Bioavailability of Isoflavones}

Bioavailability of isoflavones in humans is affected by various factors. Some of these factors are ratio of glycosidic/aglycone form, dose, source, gender, food matrix etc (Nielsen and Williamson 2007). The aglycones show better absorption than glycosidic forms in the digestive tract (Sepehr et al., 2009). Because the hydrolysis of glycosidic forms is essential for isoflavone absorption, the isoflavone aglycones are biologically active forms. Da silva et al., (2011) studied the effect of fermentation on isoflavone forms in whole soybean flour. Fermented whole soybean flour showed conversion of glycosidic forms into aglycones. Walsh et al., (2003) proposed that the isoflavones bioavailability from foods containing fat and protein is more than that of supplements due to enhanced bile secretion.
This occurs because micellarization appears to be required for bioaccessibility of isoflavone aglycones. Isoflavones upon invitro gastrointestinal digestion show high bioaccessibility which is sufficient enough to be absorbed and to impart health benefits (Rodríguez-Roque et al., 2013). Genistein and glycitein get degraded into metabolites with no apparent estrogenic activity but the daidzein may get metabolized into equol having higher estrogenic potential (Setchell et al., 2002; Simons et al., 2005).

\section{Future prospects}

Seeing the trend of malnutrition problem in India and the potential role soybean can play in addressing this problem, the opportunities are immense. The presence of phytochemicals like isoflavones can help in popularising soybased products by marketing it as a 'functional food' due its well-known health promoting effects. The biosynthetic pathway of isoflavones is well established, although lesser is known about the formation of phytoalexins from isoflavones. This can be the area of interest for plant biologists especially those working in the area of plant pathology. Bioavailability is important to achieve maximum possible health benefits from isoflavones. Using microbes either for fermentation or isolating catalytically efficient enzymes for conversion into bioactive form may be another promising area for future.

\section{References}

Akiyama T Ishida J Nakagawa S Ogawara $\mathrm{H}$ Watanabe S Itoh N Shibuya M Fukami Y (1987) Genistein, a specific inhibitor of tyrosine-specific protein kinases. The Journal of biological chemistry 262(12): 5592-5

Cassidy A, Bingham S, Setchell KD (1994) Biological effects of a diet of soy 
protein rich in isoflavones on the menstrual cycle of premenopausal women. Am J Clin Nutr 60(3): 333-40.

Chennupati P, Seguin P, Chamoun R, Jabji S (2012) Effects of high temperature stress on soybean isoflavone concentration and expression of key genes involved in isoflavone synthesis. J Agric Food Chem 60: 12421-7.

Da Silva LH, Celeghini RMS, Chang YK (2011) Effect of the fermentation of whole soybean flour on the conversion of isoflavones from glycosides to aglycones. Food Chemistry 128: 640-4.

Dastmalchi M and Dhaubhadel S (2015) Proteomic insights into synthesis of isoflavonoids in soybean seeds. Proteomics 15(10): 1646-57.

Dastmalchi M, Bernards MA, Dhaubhadel S (2016) Twin anchors of the soybean isoflavonoid metabolon: evidence for tethering of the complex to the endoplasmic reticulum by IFS and $\mathrm{C} 4 \mathrm{H}$. The Plant Journal 85: 689-706.

Dhaubhadel S, Farhangkhoee M, Chapman R (2008) Identification and characterization of isoflavonoid specific glycosyltransferase and malonyl transferase from soybean seeds. J. Exp. Bot. 59: 981-94.

Dhaubhadel S, McGarvey BD, Williams R, Gijzen M (2003) Isoflavonoid biosynthesis and accumulation in developing seeds. Pl Mol Biol 53: 73343.

Diplock AT (1994) Antioxidants and disease prevention. Molecular Aspects of Medicine. 15: 295-376.

Djuric Z, Chen G, Doerge DR, Heilbrun LK, Kucuk O (2001) Effect of soy isoflavone supplementation on markers of oxidative stress in men and women. Cancer Letters 172(1): 1-6.

Dong X, Xu W, Sikes RA, Wu C (2013) Combination of low dose of genistein and daidzein has synergistic preventive effects on isogenic human prostate cancer cells when compared with individual soy isoflavone. Food Chemistry 141: 1923-33.

Farag M, Huhman D, Dixon R, Sumner L (2008) Metabolomics reveals novel pathways and differential mechanistic and elicitor-specific responses in phenylpropanoid and isoflavonoid biosynthesis in Medicago truncatula cell cultures. Plant Physiol. 146: 387402.

Fritz H, Seely D, Flower G, Skidmore B, Fernandes R, Vadeboncoeur S, Kennedy D, Cooley K, Wong R, Sagar S, Sabri E, Fergusson D (2013) Soy, Red Clover, and Isoflavones and Breast Cancer: A Systematic Review. PLoS ONE 8(11): e81968. doi:10.1371/journal.pone.0081968.

Gutierrez-Gonzalez JJ, Guttikonda SK, Tran LP, Aldrich DL, Zhong $\mathrm{R}$, Yu O, Nguyen HT, Sleper DA (2010) Differential expression of isoflavone biosynthetic genes in soybean during water deficits. Pl Cell Physiol 51(6): 936-48.

Hoeck JA Fehr WR Murphy PA Welke GA (2000) Influence of genotype and environment on isoflavone contents of soybean. Crop Science 40(1): 48-51.

Jung W, Yu O, Lau SM, O'Keefe DP, Odell J, Fader G, Mc Gonigle B (2000) Identification and expression of isoflavone synthase, the key enzyme for biosynthesis of isoflavones in legumes. Nature Biotechnology 18(2): 208-12.

Kikuno N, Shiina H, Urakami S, Kawamoto K, Hirata H, Tanaka Y, Majid S, Igawa M, Dahiya R (2008) Genistein mediated histone acetylation and demethyla-tion activates tumor suppressor genes in prostate cancer cells. International Journal of Cancer 123(3): 552-560.

Kirakosyan A, Kaufman P, Nelson RL, Kasperbauer MJ, Duke JA, Seymour E, 
Chang SC, Warber S, Bolling S (2006) Isoflavone levels in five soybean (Glycine max) genotypes are altered by phytochrome mediated light treatments. J Agric Food Chem 54: 54-8.

Kudou S, Fleury Y, Welti D, Magnolato D, Uchida T, Kitamura K, Okubo K (1991) Malonyl isoflavone glucosides in soybean seeds. Agric. Biol. Chem. 55: 2227-33.

Kuiper GG, Carlsson B, Grandien K, Enmark E, Haggblad J, Nilsson S, Gustafsson JA (1997) Comparison of the ligand binding specificity and transcript tissue distribution of estrogen receptors alpha and beta. Endocrinology 138(3): 86370.

Kuiper GG, Lemmen JG, Carlsson B, Corton JC, Safe SH, Saag PT, Burg B, Gustafsson JA (1998) Interaction of estrogenic chemicals and phytoestogens with estrogen receptor beta. Endocrinology 139(10): 4252-63.

Kurrat A, Diel P, Blei T, Kluxen F, Mueller D, Pichotta M, Soukup S, Kulling S, Oden C (2016) Combined effects of soy isoflavones and a high fat diet on the mammary gland in an animal model of diet-induced obesity. Cancer Res 76(4 Suppl): Abstract nr P3-09-03.

Lee JH, Renita M, Fioritto RJ, Martin SK, Schwartz SJ, Vodovotz Y (2004) Isoflavone Characterisation and Antioxidant Activity of Ohio Soybeans. Journal of Agricultural and Food Chemistry 52(9): 2647-51.

Lee SJ Ahn JK Kim SH Kim JT Han SJ Jung MY Chung IM (2003) Variation in isoflavones of soybean cultivars with location and storage duration. Journal of Agricultural and Food Chemistry 51(11): 3382-9.

MacDonald RS, Guo JY, Copeland J, Browning JD, Sleper D, Rottinghaus GE, Berhow MA (2005) Environmental influences on isoflavones and saponins in soybeans and their role in colon cancer. J. Nutr. 135: 1239-42.

Majid S, Kikuno N, Nelles J, Noonan E, Tanaka Y, Kawamoto K, Hirata $\mathrm{H}, \mathrm{Li}$ LC, Zhao H, Okino ST, Place RF, Pookot D, Dahiya R (2008) Genistein induces the p21WAF1/CIP1 and p16INK4a tumor suppressor genes in prostate cancer cells by epigenetic mechanisms involving active chromatin modification Cancer Research 68 (8): 2736-44.

Nielsen ILF and Williamson G (2007) Review of the Factors Affecting Bioavailability of Soy Isoflavones in Humans. Nutrition and Cancer 57(1): $1-10$.

Pandey A, Misra P, Khan MP, Swarnkar G, Tewari MC, Bhambhani S, Trivedi R, Chattopadhyay $\mathrm{N}$ and Trivedi PK (2014) Co-expression of Arabidopsis transcription factor, AtMYB12, and soybean isoflavone synthase, GmIFS1, genes in tobacco leads to enhanced biosynthesis of isoflavones and flavonols resulting in osteoprotective activity Plant Biotechnology Journal 12: 69-80

Patel RP, Boersma BJ, Crawford JH, Hogg N, Kirk M, Kalyanaraman B, Parkes DA, Barnes S, Usmar VD (2001) Antioxidant mechanisms of isoflavones in lipid systems: Paradoxical effects of peroxyl radical scavenging. Free Radical Biol. Med. 31: 1570-81

Pratt DE and Birac PM (1979) Source of antioxidant activity of soybeans and soyproducts. J. Food Sci. 44: 1720-2

Pregelj L, McLanders JR, Gresshoff PM, Schenk PM (2010) Transcription profiling of the isoflavone phenylpropanoid pathway in soybean in response to Bradyrhizobium japonicum inoculation. Functional Plant Biology 38: $13-24$

Rice-Evans CA, Miller NJ, Paganga G (1997) 
Antioxidant properties of phenolic compounds. Trends in Plant Science 2: 152-9

Rimbach G, Pascual-Teresa SD, Ewins BA, Matsugo S, Uchida Y, Minihane AM, Turner R, Vafei AK, Weinberg PD (2003) Antioxidant and free radical scavenging activity of isoflavone metabolites. Xenobiotica 33(9): 913-25

Rodríguez-Roque MJ, Rojas-Graï MA, ElezMartínez P, Martín-Belloso O (2013) Soymilk phenolic compounds, isoflavones and antioxidant activity as affected by in vitro gastrointestinal digestion. Food Chemistry 136: 206-12

Rui HL (2004) Potential synergy of phytochemicals in cancer prevention: mechanism of action. J Nutr 134(12): 3479S-85S

Ruiz-Larrea MB, Mohan AR, Paganga G, Miller NJ, Bolwell GP, Rice-Evans CA (1997) Antioxidant activity of phytoestrogenic isoflavones. Free Radical Res 26(1): 63-70

Sarkar FH, Li Y (2003) Soy isoflavones and cancer prevention. Cancer Investigation 21(5): 744-57

Sarwar G, Peace RW, Botting HG, Brulé D (1989) Digestibility of protein and amino acids in selected foods as determined by a rat balance method. Plant Foods for Human Nutrition 39(1): 23-32

Sepehr E, Cooke GM, Robertson P, Gilani, GS (2009) Effect of glycosidation of isoflavones on their bioavailability and pharmacokinetics in aged male rats. Molecular Nutrition \& Food Research 53(Suppl 1): S16-S26

Setchell KDR Brown NM Lydeking-Olsen E (2002) The clinical importance of the metabolite equol-a clue to the effectiveness of soy and its isoflavones. The Journal of Nutrition. 132(12): 3577-84.

Shih, CH, Chen Y, Wang M, Chu IK, Lo C
(2008) Accumulation of isoflavone genistin in transgenic tomato plants overexpressing a soybean isoflavone synthase gene. J. Agric. Food Chem. 56: 5655-61.

Shinde AN, Malpathak N, Fulzele DP (2009) Enhanced production of phyto estrogenic isoflavones from hairy root cultures of Psoralea corylifolia L. Using elicitation and precursor feeding. Biotechnology and Bioprocess Engineering 14:288-94.

Simons AL, Renouf M, Hendrich S, Murphy PA (2005) Metabolism of Glycitein (7,4'-Dihydroxy-6-methoxy-isoflavone) by Human Gut Microflora. Journal of Agricultural and Food Chemistry 53(8): 8519-25.

Steele CL, Gijen MA, Qutob D, Dixon RA (1999) Molecular characterization of the enzyme catalyzing the aryl migration reaction of isoflavonoid biosynthesis in soybean. Arch Biochem. Biophys 367: 146-50.

Subramanian S, Hu X, Lu G, Odelland JT, Yu O (2004) The promoters of two isoflavone synthase genes respond differentially to nodulation and defence signals in transgenic soybean roots. Plant Molecular Biology, 54(5): 623-39.

Subramanian S, Stacey G, Yu O (2006) Endogenous isoflavones are essential for the establishment of symbiosis between soybean and Bradyrhizobium japonicum. The Plant Journal 48: 26173.

Suzuki H, Nishino T, Nakayama T (2007) cDNA cloning of a BAHD acyltransferase from soybean (Glycine max): isoflavone 7-O-glucoside-6'"-Omalonyltransferase. Phytochem. 68: 2035-42

Tanhindarto RP, Hariyadi P, Purnomo1 EH, Irawati Z (2013) Effects of gamma irradiation at different combinations of dose-rate and time of exposure on the 
isoflavone contents of soybean. As. $J$. Food Ag-Ind. 6(6): 322-8.

Tian L and Dixon RA (2006) Engineering isoflavone metabolism with an artificial bifunctional enzyme. Planta 224: 496507.

Veitch NC (2007) Isoflavonoids of the leguminosae. Nat. Prod. Rep. 24: $417-$ 64.

Walsh KR, Zhang YC, Vodovotz Y, Schwartz SJ, Failla ML (2003) Stability and Bioaccessibility of Isoflavones from Soy Bread during In Vitro Digestion. $J$. Agric. Food Chem. 51(16): 4603-9.

Wang HJ and Murphy PA (1994) Isoflavone composition of American and Japanese soybeans in Iowa: effects of variety crop, year and location. Journal of Agricultural and Food Chemistry 42(8): 1674-7

Whitehead SA Cross JE Burden C Lacey M (2002) Acute and chronic effects of genistein, tyrphostin and lavendustin $\mathrm{A}$ on steroid synthesis in luteinized human granulosa cells. Human Reproduction 17(3): 589-94.

Wiseman H O'Reilly JD Adlercreutz H Mallet AI Bowey EA Rowland IR Sanders TA (2000) Isoflavone phytoestrogens consumed in soy decrease $F(2)$ isoprostane concentrations and increase resistance of low-density lipoprotein to oxidation in humans. American Journal of Clinical Nutrition 72(2): 395-400.

$\mathrm{Xu}$ B and Chang SK (2008) Characterization of phenolic substances and antioxidant properties of food soybeans grown in the North Dakota-Minnesota Region. Journal of Agricultural and Food Chemistry. 56(19): 9102-13.

Yu O, Jung W, Shi J, Croes RA, Fader GM, McGonigle B, Odell JT (2000) Production of the isoflavones genistein and daidzein in non-legume dicot and monocot tissues. Plant Physiol. 124: $781-94$.

\section{How to cite this article:}

Sandeep Kumar. 2017. Isoflavones: Biosynthesis, Health Promoting Effects and Bioavailability. Int.J.Curr.Microbiol.App.Sci. 6(12): 1062-1071. doi: https://doi.org/10.20546/ijcmas.2017.612.120 